\title{
SUBSTRUCTURES IN THE NARROW-LINE REGION OF AGN
}

\author{
Sylvain Veilleux \\ Lick Observatory \\ University of California \\ Santa Cruz, CA 95064 \\ USA
}

\begin{abstract}
The preliminary results of a high-resolution study of narrow emission-line profiles in bright Seyfert galaxies are reported. Profile substructures such as secondary peaks and shoulders are observed in nearly all the objects. The results of profile comparisons in Mrk 359, Mrk 533, and Mrk 1066 are briefly discussed.
\end{abstract}

\section{Observations}

The Hamilton echelle spectrograph installed at the coudé focus of the Shane 3-m telescope was used to obtain high signal-to-noise spectra of Seyfert galaxies covering the $4600 \AA$ $7500 \AA$ at an average resolution of about $10 \mathrm{~km} \mathrm{sec}^{-1}$. Fourteen galaxies have been observed so far. These galaxies were selected on the basis of their apparent magnitude and the quality of their optical spectrum (numerous stong emission lines, weak stellar absorption lines, ...). The data were reduced using the method discussed in Goodrich and Veilleux (1988). The usual line profile parameters (peak and center velocities, widths, asymmetries, and area parameters) have been measured for all the galaxies. The excellent resolution of the data allowed the measurement of these parameters in the very core of the lines. Profile comparison ("velocity mapping") was done for a number of galaxies.

\section{Results}

Comparisons of the results of the present study with the results of Vrtilek and Carleton (1984) generally show very good agreement for both the widths and line center velocities of [O III] $\lambda 5007$. On the other hand, line width measurements of lower resolution studies tend to be larger than the present measurements. The discrepancy appears to be a function of the resolution, being larger for narrower emission lines and lower resolution studies. Note, however, that the line centroids are essentially unaffected by resolution effects.

One of the major results of the present study is that nearly all of the galaxies observed to date show clear evidence of profile substructures in their narrow emission lines. Secondary peaks are seen in the line profiles of a number of galaxies. In some galaxies the subcomponents are located in the wings of the line profiles while in others they constitute the core of the emission lines. 


\section{Discussion of Mrk 359, Mrk 533, and Mrk 1066}

Mrk 359 is somewhat atypical of the galaxies observed in the present study since no profile substructure is found in its emission lines. The emission lines of Mrk 359 with low ionization potential/critical density are very narrow (FWHM $\sim 100-200 \mathrm{~km} \mathrm{sec}^{-1}$ ), and slightly blue asymmetric. On the hand, lines with high ionization/critical density are broader, highly blue asymmetric, and their peaks are blueshifted. Comparison of the density-sensitive lines [S II] $\lambda \lambda 6716,6731$ indicates that the average density of the gas producing the blue side of these lines may be lower than the average density of the gas producing the red side. This is opposite to the predictions of the NLR models which include density stratification.

All of the emission lines of Mrk 533 (NGC 7674) present very strong blue wings sometimes extending out to $\mathrm{v} \lesssim-2000 \mathrm{~km} \mathrm{sec}^{-1}$ with respect to the peak. A small secondary peak is observed at $\mathrm{v}=(-250,-300)$ in both $\mathrm{H} \alpha$ and [N II]. A comparison of $\mathrm{H} \alpha$ with [O III] $\lambda 5007$ indicates that the $[\mathrm{O} \mathrm{III}] / \mathrm{H} \alpha$ ratio is higher in the wings than in the main and blueshifted components. The intensity of the blue component may be affected by aperture effects but not the blue wing. These results suggest that the extended blue wing is produced by high-velocity outflowing gas located relative close to the nucleus and characterized by a high ionization parameter while the blue component is characterized by a lower ionization parameter, is located further out from the nucleus, and is outflowing at a lower velocity. The detection of a blueshifted H I absorption line by Mirabel (1982) is consistent with this idea. Since the [S II] lines are badly affected by the atmospheric B band it is impossible to compare the density of the gas in the components with the gas producing the wings. Perhaps there is a connection between the components observed in the optical spectra and the double radio structure observed by Unger et al. (1986).

All of the emission lines observed in Mrk 1066 (NGC 4253) show the presence of two components in the core of the lines separated by about $120 \mathrm{~km} \mathrm{sec}^{-1}$. Aperture effects affecting the relative intensities of these components suggest that the blue component is produced by material further away from the continuum nucleus than the blue wing and the red component. Comparison of the emission line profiles of $\mathrm{H} \alpha$, [N II], [S II], and [O III] obtained simultaneously indicates that the blue component is of higher ionization level than the red component but still produces appreciable amount of [N II] and [S II] emission. The reason for the higher ionization level in the blue component is not clear at the moment; perhaps the blue component is photoionized by a partially beamed ionizing continuum coming out of the nucleus or the source of ionization is in or near the component itself. Line ratio measurements $([\mathrm{O} \mathrm{III}] / \mathrm{H} \beta,[\mathrm{N} \mathrm{III}] / \mathrm{H} \alpha$, etc.) are needed to correctly address this question. The possible detection of a double structure in the [O III] image of this object by Haniff et al. (1988) is consistent with the present results.

\section{References}

Goodrich, R. W., and Veilleux S. 1988, Publ. A. S. P., in press.

Haniff, C. A., Wilson, A. S., and Ward, M. J. 1988, Ap.J., in press.

Mirabel, I. F. 1982, Ap. J., 260, 75.

Ulvestad, J. S., and Wilson, A. S. 1984, Ap. J., 278, 544.

Unger, S. W., Pedlar, A., Booler, R. V., and Harrison, B. A. 1986, M.N.R.A.S., 219, 387.

Vrtilek, J. M., and Carleton N. P. 1984, Ap. J., 294, 106. 